\title{
IMPLEMENTATION OF ENVIRONMENTAL MANAGEMENT SYSTEM IN THE BRAZILIAN ANTARCTIC BASE
}

http://dx.doi.org/10.4322/apa.2014.050

\author{
Alexandre de Avila Leripio ${ }^{1, *}$, Fernanda Helena Leite ${ }^{2}$, Rafaela Picolotto ${ }^{1}$, Mariana de Sá Viana ${ }^{2}$ \\ 'Laboratório de Sistema de Gestão Ambiental, Universidade do Vale do Itajaí - UNIVALI, Itajaí, SC, Brazil \\ ${ }^{2}$ Ministério do Meio Ambiente, Esplanadas dos Ministérios, Bloco B, Brasilia, Brazil \\ *e-mail: sgaeacf@gmail.com
}

In 2009 the Brazilian Antarctica Programme (well known acronym, PROANTAR in Brazilian-Portuguese) decided to implement the Environmental Management System - EMS certifiable through the international norm NBR ISO14001:2004 at Comandante Ferraz Antarctic Base (acronym - EACF in Brazilian-Portuguese). This initiative is being undertaken with the coordination of the Ministry of the Environment and with the participation of the National Institute for Science and Technology - Antarctic Environmental Research - (acronym in Brazilian Portuguese - INCT-APA) in strict contact with the Secretariat of the Interministerial Commission for the Resources of the Sea - (acronym SECIRM in BrazilianPortuguese) coordinated by the Brazilian Navy, which is the organization responsible for the logistical, administrative and organizational management of EACF.

Since EACF is located in such a unique environment as the Antarctica, with high environmental sensibility, and in accordance with commitments assumed by the Brazilian Government in the Madrid Protocol, the activities undertaken at the Brazilian Base should follow procedures so as to cause the least possible environmental impact. For this purpose, the controls established focus on preventative actions related to its activities.

EACF consists of several modules in which are distributed, lodgings, laboratories, workshops, sitting rooms, infirmary, kitchen, bakery, freezers, library, storage area, communication room, a small sports gym (academy) and research laboratories. Its fixed population is made up of 15 military personnel from the Brazilian Navy who are based at $\mathrm{EACF}$ for a 1 year long period, being relieved in two stages, in March/April and October/November and furthermore by members of the Navy Dockyard Personnel from Rio de Janeiro - (AMRJ, Portuguese acronym), responsible for the refurbishments, expansions and structural maintenance of the base. The floating population consists of approximately 50 people lodged at the Base in summer, the majority researchers. The floating population varies, in that each group has a permanence period of approximately 30 days.

Considering the fragility of the Antarctic environment and the potential environmental impacts associated to the rotation of researchers and to the routine activities undertaken at EACF, a system of control of the environmental aspects becomes essential. The Environmental Management System is structured in order to manage and minimize impacts that occur as a consequence of human activity.

According to NBR ISO 14001:2004, the environmental aspect is defined thus:

"[...] it is the service, activity or product of an organization that can interfere in the environment, and environmental impact is any modification to the environment, adverse or beneficial, resulting from environmental aspects." (p. 4).

NBR ISO 14001:2004 requires that the organization establishes and maintains an up-to-date procedure to identify the environmental aspects of its activities and services. However, not all the aspects have to be considered, only those that were considered significant and over which the organization is able to exert control or influence.

In January 2009, a team of specialists in EMS went to EACF to undertake a survey of aspects and impacts of environmental activities that occur at EACF. The team also carried out a preliminary diagnostic of the 


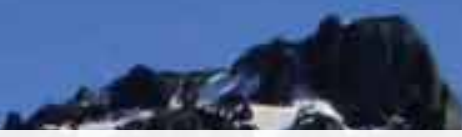

level of compliance with the requirements of norm NBR ISO 14001:2004. The results obtained permit the preliminary conclusion that there is partial compliance with the norm $-42.3 \%$ on average of compliance with norm requirements, which can be considered promising, since there is still no EMS formally implemented at this point in time.

The verification of environmental aspects and impacts was carried out at EACF during the period, February to March 2010, being validated in a specific meeting for this purpose undertaken at EACF with participation of representatives of the several interested parties (stakeholders). Amongst the participating institutions, attention can be called to: the Brazilian Antarctica Programme (PROANTAR), represented by the Head of EACF and the AMRJ Engineer, the Ministry of the Environment, INCT- APA, apart from support technical staff, characterising a multi-disciplinary group. After the validation at EACF, a preliminary version of the "Survey of Environmental Aspects and Impacts of EACF" was prepared and the initial scope of EMS focused on EACF was delineated including the activities directly related to the routine, maintenance and research carried out at the Base.

In July 2010 a meeting was held in Rio de Janeiro with the purpose of identifying the necessities of the interested parties associated to the environmental aspects and impacts verified at EACF and defining the significant environmental aspects and impacts associated to the activities. The participants represented the same institutions present at the first meeting held at EACF, however, with a higher number of participants at the meeting in Rio de Janeiro.

The Base-Group of the Brazilian Navy, as already mentioned, is substituted in March of each year, and remains in training for approximately 6 months, following a rigorous selection process, which is undertaken starting in the first semester of the previous year to their going to EACF. For this reason, in order to implement EMS, the training of the Base-Group is essential, to help with the necessary implementation process and afterwards with the maintenance of the System.
Amongst the initiatives undertaken in the sense of preparing the Base-Group for the implementation of EMS are: the Course of Reading and Interpretation of norm NBR ISO 14.001:2004 and the Course of Formation of Environmental Auditors according to NBR ISO 19.011:2002.

In February of 2010, the Base-Group were given their first training concerning EMS, specifically the Course of Reading and Interpretation of norm NBR ISO 14.001:2004, which took place on 18 and 19 February 2010, at the Naval Supply Base in Rio de Janeiro with the participation of 13 military personnel and 1 representative of the Ministry of the Environment.

During the course questions related to the basic concepts of environmental management, cycles of continuous improvement which encompasses planning, implementation, control and the action, known as PDCA - plan-do-check-act, were covered.

During the course explanations were given concerning the preliminary verification of the environmental impacts and related aspects concerning EACF and in addition the EMS implementation plan was presented.

In order to verify the level of knowledge acquired concerning the norm, at the end of the last day, a written evaluation concerning the main concepts presented during the course was applied.

In August 2010, the Pre-Deployment Antarctic Training - PAT was held at the Ilha de Marambaia (Marambaia Island) in Rio de Janeiro. The purpose of the training is to prepare the Base-Group of the Navy and researchers who go to Antarctica in order to enable adaptation to the adverse conditions of the environment in which they are subject to for a good period of time. Lectures on security, first-aid, climbing, nutrition, physical conditioning and the environment were given. The mains lines of research which are being carried out in Antarctica are also presented.

During the Pre-Deployment Antarctica Training 2010, a lecture for increasing conscientiousness and awareness towards EMS was delivered; it covered the aspects and impacts related to the environment raised in the last Antarctica operation and the importance of the System 
in the activities undertaken at the Base and its future certification according to norm NBR ISO 14.001:2004.The main purpose of the activity was to present EMS to new Brazilian "candidates to Antarctica", in order to obtain right from the start their commitment to the EMS objectives.

Certification process according to norm NBR ISO 14.001:2004 is initially planned for the month of January 2012 beginning with the audit by the certifying body. This stage will require special attention as regards transport logistical procedures and the permanence of the auditing team.

Through this initiative, it is hoped to fortify the actions which the Brazilian Antarctic Programme has been seeking to take ever since its creation, with the purpose of protection of the Antarctic environment, andpursing the improvement of its procedures for better compliance of what is established in the Madrid Protocol. 